\title{
Front Matter: Volume 7250
}

, "Front Matter: Volume 7250," Proc. SPIE 7250, Digital Photography V, 725001 (20 January 2009); doi: 10.1117/12.822097

SPIE Event: IS\&T/SPIE Electronic Imaging, 2009, San Jose, California, United SPIE. States 


\section{PROCEEDINGS \\ IS\&T/SPIE \\ Electronio \\ Imaging \\ Science and Technology}

\section{Digital Photography V}

Brian G. Rodricks

Sabine E. Süsstrunk

Editors

19-20 January 2009

San Jose, California, USA

Sponsored and Published by

IS\&T-The Society for Imaging Science and Technology

SPIE 
The papers included in this volume were part of the technical conference cited on the cover and title page. Papers were selected and subject to review by the editors and conference program committee. Some conference presentations may not be available for publication. The papers published in these proceedings reflect the work and thoughts of the authors and are published herein as submitted. The publishers are not responsible for the validity of the information or for any outcomes resulting from reliance thereon.

Please use the following format to cite material from this book:

Author(s), "Title of Paper," in Digital Photography V, edited by Brian G. Rodricks, Sabine E. Süsstrunk, Proceedings of SPIE-IS\&T Electronic Imaging, SPIE Vol. 7250, Article CID Number (2009).

ISSN 0277-786X

ISBN 9780819475008

Copublished by

SPIE

P.O. Box 10, Bellingham, Washington 98227-0010 USA

Telephone +1 3606763290 (Pacific Time) · Fax +1 3606471445

SPIE.org

and

IS\&T-The Society for Imaging Science and Technology

7003 Kilworth Lane, Springfield, Virginia, 22151 USA

Telephone +1 7036429090 (Eastern Time) · Fax +1 7036429094

imaging.org

Copyright (c) 2009, Society of Photo-Optical Instrumentation Engineers and The Society for Imaging Science and Technology.

Copying of material in this book for internal or personal use, or for the internal or personal use of specific clients, beyond the fair use provisions granted by the U.S. Copyright Law is authorized by the publishers subject to payment of copying fees. The Transactional Reporting Service base fee for this volume is $\$ 18.00$ per article (or portion thereof), which should be paid directly to the Copyright Clearance Center (CCC), 222 Rosewood Drive, Danvers, MA 01923. Payment may also be made electronically through CCC Online at copyright.com. Other copying for republication, resale, advertising or promotion, or any form of systematic or multiple reproduction of any material in this book is prohibited except with permission in writing from the publisher. The CCC fee code is 0277-786X/09/ $\$ 18.00$.

Printed in the United States of America.

Paper Numbering: Proceedings of SPIE follow an e-First publication model, with papers published first online and then in print and on CD-ROM. Papers are published as they are submitted and meet publication criteria. A unique, consistent, permanent citation identifier (CID) number is assigned to each article at the time of the first publication. Utilization of CIDs allows articles to be fully citable as soon they are published online, and connects the same identifier to all online, print, and electronic versions of the publication. SPIE uses a six-digit CID article numbering system in which:

- The first four digits correspond to the SPIE volume number.

- The last two digits indicate publication order within the volume using a Base 36 numbering system employing both numerals and letters. These two-number sets start with $00,01,02,03,04,05$, $06,07,08,09,0 A, O B \ldots 0 Z$, followed by 10-1Z, 20-2Z, etc.

The CID number appears on each page of the manuscript. The complete citation is used on the first page, and an abbreviated version on subsequent pages. Numbers in the index correspond to the last two digits of the six-digit CID number. 


\section{Contents}

vii Conference Committee

SENSOR DESIGN

725003 Very-large-area CCD image sensors: concept and cost-effective research [7250-39] E. W. Bogaart, I. M. Peters, A. C. M. Kleimann, E. J. P. Manoury, W. Klaassens, W. T. F. M. de Laat, DALSA Professional Imaging (Netherlands); C. Draijer, DALSA Digital Imaging (Canada); R. Frost, DALSA Semiconductor (Canada); J. T. Bosiers, DALSA Professional Imaging (Netherlands)

725004 Decoupling photo collection efficiency and color crosstalk from the quantum efficiency spectrum for the CMOS image sensor pixel development [7250-11]

Y. Wu, P. Cizdziel, H. Rhodes, OmniVision Technologies, Inc. (United States)

725005 Microlens performance limits in sub-2 $\mu \mathrm{m}$ pixel CMOS image sensors [7250-35]

Y. Huo, C. C. Fesenmaier, P. B. Catrysse, Stanford Univ. (United States)

\section{COLOR SENSING}

725006 Sensor information capacity and spectral sensitivities [7250-23]

F. Cao, F. Guichard, H. Hornung, L. Masson, DxO Labs. (France)

725007 2PFC image sensors: better image quality at lower cost [7250-42]

D. J. Tweet, J.-J. Lee, J. M. Speigle, Sharp Labs. of America (United States);

D. Tamburrino, Sharp Labs. of America (United States) and Ecole Polytechnique Fédérale de Lausanne (Switzerland)

725008 Interleaved imaging: an imaging system design inspired by rod-cone vision [7250-32] M. Parmar, B. A. Wandell, Stanford Univ. (United States)

725009 Optimal color filter array design: quantitative conditions and an efficient search procedure [7250-40]

Y. M. Lu, M. Vetterli, Swiss Federal Institute of Technology (Switzerland)

\section{COLOR PROCESSING}

7250 OA New architectures of an image-processing pipeline based on nonlinear image decomposition [7250-01]

T. Saito, D. Yamada, H. Aizawa, T. Komatsu, Kanagawa Univ. (Japan)

$7250 \mathrm{OB} \quad$ An image-noise filter with emphasis on low-frequency chrominance noise [7250-13]

R. Gheorghe, S. Goma, M. Aleksic, Advanced Micro Devices (Canada) 
7250 OC False colors removal on the YCrCb color space [7250-19]

V. Tomaselli, M. Guarnera, G. Messina, STMicroelectronics (Italy)

7250 OD Automatic white balance: whitebalPR using the dichromatic reflection model [7250-04] M. Sajjaa, G. Fischer, Cologne Univ. of Applied Sciences (Germany)

\section{SYSTEM ANALYSIS}

$7250 \mathrm{OE} \quad$ Illuminant estimation and detection using near-infrared [7250-33]

C. Fredembach, S. Süsstrunk, Ecole Polytechnique Fédérale de Lausanne (Switzerland)

7250 OF Naturalness classification of images into DCT domain [7250-09]

S. Battiato, G. M. Farinella, G. Gallo, E. Messina, Univ. degli Studi di Catania (Italy)

7250 OG Effects of imaging lens f-number on sub-2 $\mu \mathrm{m}$ CMOS image sensor pixel performance [7250-36]

C. C. Fesenmaier, Y. Huo, P. B. Catrysse, Stanford Univ. (United States)

$7250 \mathrm{OH} \quad$ Measuring texture sharpness of a digital camera [7250-21]

F. Cao, F. Guichard, H. Hornung, DxO Labs. (France)

7250 Ol Interaction of image noise, spatial resolution, and low contrast fine detail preservation in digital image processing [7250-25]

U. Artmann, D. Wueller, Image Engineering (Germany)

$7250 \mathrm{~J} \quad$ Visual model of human blur perception for scene adaptive capturing [7250-20] S.-S. Kim, D.-S. Chung, B.-K. Park, J.-B. Kim, S.-D. Lee, Samsung Advanced Institute of Technology (Republic of Korea)

MOBILE IMAGING

7250 OK Mobile imaging: the big challenge of the small pixel [7250-34]

F. Xiao, Fairchild Imaging (United States); J. Farrell, ImagEval Consulting LLC (United States) and Stanford Univ. (United States); P. Catrysse, Stanford Univ. (United States); B. Wandell, ImagEval Consulting LLC (United States) and Stanford Univ. (United States)

$7250 \mathrm{OL} \quad$ Reduction of motion blur for handheld captured images by joint stabilization and spatio-temporal denoising [7250-07]

A. Castorina, G. Spampinato, A. Bruna, A. Capra, STMicroelectronics (Italy)

7250 ON Extended depth-of-field using sharpness transport across color channels [7250-24]

F. Guichard, H. P. Nguyen, R. Tessières, M. Pyanet, I. Tarchouna, F. Cao, DxO Labs. (France) 
725000 People first: separating people from background in digital photographs [7250-03]

M. Ciuc, Tessera România (Romania) and Univ. Politehnica Bucureşti (Romania);

A. Capătă, Tessera România (Romania); A. Zamfir, Tessera România (Romania);

A. F. Drîmbărean, Tessera Ireland (Ireland); E. Steinberg, Tessera (United States)

$7250 \mathrm{OP} \quad$ Automatic background generation from a sequence of images based on robust mode estimation [7250-41]

D. Sidibé, O. Strauss, LIRMM, CNRS, Univ. of Montpellier II (France); W. Puech, LIRMM, CNRS, Univ. of Montpellier II (France) and Univ. of Nimes (France)

\section{INTERACTIVE PAPER SESSION}

7250 OR Nonlinear color-image decomposition for image processing of a digital color camera [7250-02]

T. Saito, H. Aizawa, D. Yamada, T. Komatsu, Kanagawa Univ. (Japan)

7250 OS Measuring the spectral response with a set of interference filters [7250-08]

C. Maver, D. Wueller, Image Engineering (Germany)

7250 OT Fuzzy-based motion estimation for video stabilization using SIFT interest points [7250-10] S. Battiato, G. Gallo, G. Puglisi, Univ. of Catania (Italy); S. Scellato, Scuola Superiore di Catania (Italy)

7250 OU Chromatic aberration reduction through optical feature modeling [7250-16]

J. Kang, H. Ok, J. Lim, S.-D. Lee, Samsung Electronics Co., Ltd. (Republic of Korea)

7250 OV Exposure-adaptive color-image enhancement [7250-27]

J.-H. Kwon, W. Choe, K. Lee, S.-D. Lee, Samsung Advanced Institute of Technology (Republic of Korea)

7250 OW Statistical identification and analysis of defect development in digital imagers [7250-29] J. Leung, G. H. Chapman, Simon Fraser Univ. (Canada); Z. Koren, I. Koren, Univ. of Massachusetts, Amherst (United States)

7250 OX IDEAL: an image pre-processing architecture for high-end professional DSC applications [7250-38]

A. van der Heide, T. Urano, F. Polderdijk, W. de Haan, J. T. Bosiers, DALSA Professional Imaging (Netherlands)

7250 OY Fusion of high dynamic range scene photos [7250-44]

E. V. Tolstaya, M. N. Rychagov, Samsung Research Ctr. (Russian Federation); K. Kang,

S. H. Kim, Samsung Electronics Co., Ltd. (Republic of Korea)

$7250 \mathrm{OZ}$ Retargeting of digital images and documents [7250-45]

V. Bucha, I. Safonov, M. Rychagov, Samsung Research Ctr. (Russian Federation); J. K. Hong,

S. H. Kim, Samsung Electronics Co., Ltd. (Republic of Korea) 
Downloaded From: https://www.spiedigitallibrary.org/conference-proceedings-of-spie on 25 Apr 2023

Terms of Use: https://www.spiedigitallibrary.org/terms-of-use 


\title{
Conference Committee
}

\author{
Symposium Chairs
}

Nitin Sampat, Rochester Institute of Technology (United States)

Jan P. Allebach, Purdue University (United States)

Conference Chairs

Brian G. Rodricks, Fairchild Imaging (United States)

Sabine E. Süsstrunk, École Polytechnique Fédérale de Lausanne

(Switzerland)

Program Committee

Donald J. Baxter, STMicroelectronics (United Kingdom)

Peter B. Catrysse, Stanford University (United States)

Ted J. Cooper, Foveon, Inc. (United States)

Jeffrey M. DiCarlo, Hewlett-Packard Laboratories (United States)

Edward R. Dowski, Jr., Omnivision CDM Optics, Inc. (United States)

Joyce E. Farrell, Stanford University (United States)

Boyd A. Fowler, Fairchild Imaging (United States)

Frederic Guichard, DxO Laboratories (France)

Francisco Imai, Samsung Information Systems America, Inc. (United States)

Michael A. Kriss, Consultant (United States)

Jingqiang Dylan Li, LifeSize Communications, Inc. (United States)

Russel A. Martin, Foveon, Inc. (United States)

Kevin J. Matherson, Hewlett-Packard Company (United States)

Seishi Ohmori, Nikon Corporation (Japan)

Gloria G. Putnam, Eastman Kodak Company (United States)

John R. Reinert-Nash, Lifetouch, Inc. (United States)

Todd Sachs, Aptina Imaging (United States)

Nitin Sampat, Rochester Institute of Technology (United States)

Dietmar Wueller, Image Engineering (Germany)

Feng Xiao, Fairchild Imaging (United States)

\section{Session Chairs}

1 Computational Photography

Brian G. Rodricks, Fairchild Imaging (United States)

Sabine E. Süsstrunk, Ecole Polytechnique Fédérale de Lausanne (Switzerland) 
2 Sensor Design

Peter B. Catrysse, Stanford University (United States)

3 Color Sensing

Donald J. Baxter, STMicroelectronics Ltd. (United Kingdom)

$4 \quad$ Color Processing

Michael A. Kriss, Consultant (United States)

5 System Analysis

Francisco H. Imai, Samsung Information Systems America, Inc. (United States)

$6 \quad$ Mobile Imaging

Feng Xiao, Fairchild Imaging (United States)

7 Computational Photography

Frédéric Guichard, DxO Laboratories (France) 